\title{
Effect of Facet Joint Effusion on Clinical and Radiographic Outcomes of Decompression for Degenerative Lumbar Spinal Stenosis
}

\author{
Jae Yeon Kim¹, Jung Hwan Lee, ${ }^{2}$ Seung Chan Yoo', Chung Kee Chough ${ }^{1 凶}$ \\ 'Department of Neurosurgery, Yeouido St. Mary's Hospital, College of Medicine, The Catholic University of Korea, Seoul, \\ ${ }^{2}$ Department of Neurosurgery, Pusan National University Hospital, Pusan National University School of Medicine, Busan, Korea
}

\begin{abstract}
Objective: The purpose of this study was to investigate the effect of facet joint effusion without radiographic instability on clinical and radiographic outcomes of decompression for degenerative lumbar spinal stenosis (DLSS). Methods: We analyzed consecutive patients with DLSS undergoing L4-L5 decompression from May 2007 to January 2017 at a single institution. We investigated the effect of facet joint effusion on clinical and radiographic outcomes. For clinical assessment, visual analogue scale (VAS) for back and leg pain and Oswestry disability index (ODI) were measured. Slip percentage and segmental motion were measured on standing lateral lumbar flexion-extension (SLFE) radiographs. Facet joint effusion was measured on axial MRI for radiographic assessment. Results: Facet joint effusion was not associated with VAS score for back pain, VAS score for leg pain, or ODI score at 2 years postoperatively $(p=0.317, p=0.159$, and $p=0.054$, respectively). Facet joint effusion was not associated with postoperative segmental motion at the index level $(p=0.953)$. However, there was a significant association between facet joint effusion and postoperative slip percentage $(p=0.014)$. Conclusion: Facet joint effusion without radiographic instability has no effect on the clinical outcomes of decompression for DLSS except for postoperative slip percentage. Progression instability was very rare.
\end{abstract}

Key Words: Decompression; Facet effusion; Instability; Lumbar spinal stenosis; Spinal fusion

$\triangle$ Corresponding Author: Chung Kee Chough, Department of Neurosurgery, Yeouido St. Mary's Hospital, College of Medicine, The Catholic University of Korea, 10,63-ro, Yeongdeungpo-gu, Seoul 07345, Korea. Tel: +82-2-3779-1187, Fax: +82-2-786-5809, E-mail: chough@catholic.ac.kr

\section{INTRODUCTION}

Degenerative lumbar spinal stenosis (DLSS) is one of the most common surgically treated spinal disorders. Generally, there are two main surgical methods to treat DLSS: (1) decompression and (2) decompression plus fusion. The addition of fusion to decompression in DLSS surgery depends on the existence of preoperative segmental instability ${ }^{10,13)}$. Traditionally, anterior-posterior translation measured on standing lateral lumbar flexion-extension (SLFE) radiographs has been regarded as the standard for evaluating segmental instability ${ }^{14)}$. However, this method has its own limitations and problems ${ }^{1,7}$. Therefore, a lot of efforts have been made to find more reliable test methods to accurately identify preoperative segmental instability.

Recently, several studies have suggested that facet joint effusion detected on magnetic resonance imaging (MRI) is indicative of segmental instability and that the presence of facet joint effusion and radiographic instability may predict an increased likelihood of achieving good surgical outcome following fusion surgery ${ }^{2,3,19)}$.
However, facet joint effusion is observed even in the absence of radiographic instability, and there are few reports on clinical and radiographic significance of this situation. Thus, the purpose of this study was to investigate the effect of facet joint effusion without radiographic instability on clinical and radiographic outcomes of decompression for DLSS.

\section{MATERIAL AND METHODS}

\section{Patients}

We performed a retrospective review of prospectively collected data. Patients with symptomatic DLSS without radiographic instability who failed more than 3 months of conservative treatment were considered as candidates for minimally invasive lumbar decompression surgery. Radiographic instability was evaluated using dynamic radiographs. The absence of instability was defined by the following three criteria: $<25 \%$ slip of L4-L5 in neutral position, $<3 \mathrm{~mm}$ in translation between flexion and extension bending, and $<5$ degrees of local kyphosis angle 
in flexion position ${ }^{11)}$.

The surgical procedure was almost identical to that described by Hatta et al. ${ }^{9)}$ as follows.. For L4-L5 decompression, a 30-mm midline skin incision was made, centered at the L4-L5 interspinous level. The muscles and periosteum were detached together from the bone. The caudal half of the L4 spinous process/lamina and the cranial third of the L5 spinous process/ lamina were exposed. A surgical microscope was used after exposing the L4-L5 interlaminar space. The surgical field was gradually expanded by retracting the split ligaments and bilateral paravertebral muscles laterally using a Gelpi self-retaining retractor. After removing the caudal part of the L4 spinous processes, the cranial third of the L5 lamina was removed to free the caudal margin of the ligamentum flavum. After partial laminotomy of the caudal half of the L4 lamina, a dome-like expansion was performed by removing the inner laminar plate to the extent where the cranial margin of the ligamentum flavum was freed. The bilateral facet joint was undercut to expose the lateral margin of the ligamentum flavum. The ligament was then removed using a pituitary punch or a Kerrison rongeour. The central canal and nerve root canal decompressions were accomplished by trimming the medial margins of the upper and lower articular processes together with the capsular portion of the ligamentum flavum flush with the medial border of the pedicle bilaterally. During the surgery, an utmost effort was made to preserve the facet joint integrity. Inclusion criteria for this study were as follows: (1) one-level L4-L5 DLSS undergoing a decompression, (2) preoperative MRI and dynamic radiographs, (3) postoperative dynamic radiograph, and (4) a minimum of two-year follow-up of clinical and radiographic data. Exclusion criteria were as follows: (1) history of previous spine surgery, (2) additional spine surgery during follow-up, and (3) postoperative infection. From May 2007 to January 2017, a total of 249 patients underwent decompression for DLSS. Among them, 188 patients underwent multilevel decompression or decompression at levels other than L4-L5 or had follow-up of less than two years. Finally, a total of 31 cases fulfilled our inclusion criteria. Demographic data of these subjects are summarized in Table 1.

\section{Clinical and Radiographic Outcomes}

Clinical outcomes were evaluated with the visual analog scale (VAS, range: 0-10, with 0 reflecting no pain) and the Oswestry

Table 1. Demographic characteristics of subjects

\begin{tabular}{lc}
\hline \hline Characteristics & Totals \\
\hline Number of patients & 31 \\
Age (years) & $69.4(58-80)$ \\
Sex (male/female) & $12 / 19$ \\
FU periods (months) & $39(29-58)$ \\
\hline
\end{tabular}

disability index (ODI).

Radiographic outcomes were evaluated preoperatively and at two years after surgery with SLFE radiographs. The distance between the posterior end of the inferior endplate of L4 and the superior endplate of L5 was divided by sagittal width of the superior endplate of L5 body to calculate a slip percent (Fig. 1). L4-L5 segmental motion was assessed by measuring the difference between the slip distance on the standing flexion versus extension lumbar radiographs (Fig. 2).

Facet joint effusion was defined as a curvilinear, measurable, high intensity signal within the facet joint that closely matched that of cerebrospinal fluid. The maximal distance between the superior and inferior articular processes at the L4-L5 facet was measured on preoperative axial T2-weighted images (Fig. 3).

These radiographic data were measured using a measuring program with a built-in picture archiving communication system (PACS, Maroview; Marotech Co, Seoul, Korea).

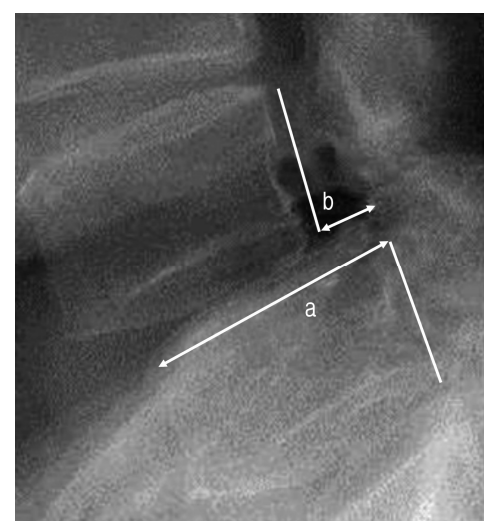

Fig. 1. Lateral lumbar radiograph demonstrating slip percent measurement. a: Sagittal width of the superior end plate of L-5 vertebra, b: Slip distance, slip percentage is b/aX100 (\%).

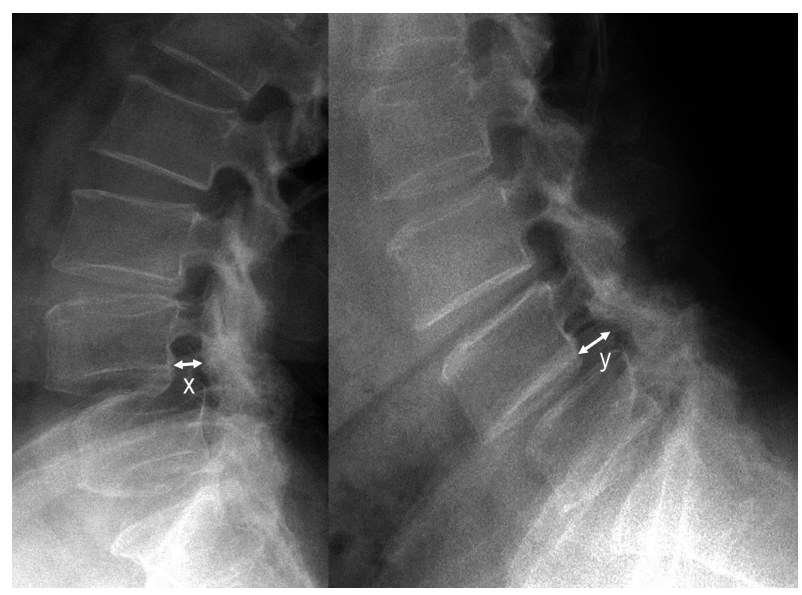

Fig. 2. Segmental motion measurement method. $x$ : Slip distance on extension lateral lumbar radiograph; y: slip distance on flexion lateral lumbar radiograph. Segmental motion is $y^{-} x(\mathrm{~mm})$ 


\section{Statistical analysis}

Clinical and radiographic data were analyzed using the PASW 18.0 statistics software (SPSS Inc. Chicago, IL, USA). Spearman's correlation coefficient analysis, Student's t-test, and paired t-test were used. A probability value of less than 0.05 was considered significant.

\section{RESULTS}

Clinical parameters were summarized in Table 2. Before surgery, all patients had moderate or severe pain scores. After two years, these scores were significantly decreased. Preoperative and postoperative slip percentage or segmental motion were similar to each other.

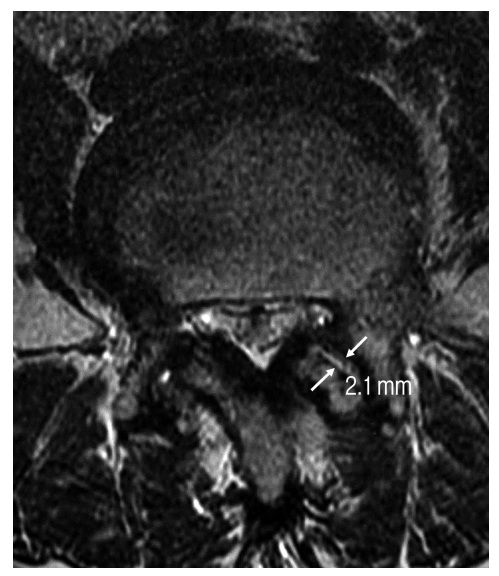

Fig. 3. Facet joint effusion measurement method on axial T2weighted image. Maximal distance between the superior and inferior articular processes where the effusion signal matches the signal of cerebrospinal fluid.
No significant association was found between the preoperative slip percentage and postoperative clinical outcomes. Preoperative segmental motion did not affect postoperative clinical outcomes either. However, the amount of facet joint effusion was weakly correlated with postoperative ODI scores without reaching statistically significant level (Spearman's rank correlation coefficient $=0.355, p=0.054$ ) (Table 3).

There were no significant associations between the facet joint effusion and pre- or post-operative radiographic parameters except for postoperative slip percentage ( $p=0.014)$. The amount of the facet joint effusion increased postoperative slip percentage. However, the increased slip percentage after surgery did not affect the clinical outcome.

\section{DISCUSSION}

Whether additional spinal fusion is necessary for surgical treatment of DLSS remains controversial ${ }^{17}$. In general, it has been considered as an indication of the addition of spinal fusion to decompression in patients with severe discogenic low back pain, foraminal stenosis, spinal deformity or instability ${ }^{5,17,18)}$. Among these, segmental instability is the most widely accepted and important indication of the addition of spinal fusion. Therefore, accurate evaluation and judgment of the presence of segmental instability before surgery are important to determine whether spinal fusion is necessary.

Traditionally, anterior-posterior translation measured on SLFE radiographs has been regarded as the gold standard for evaluating segmental instability ${ }^{14}$. Patients were considered to have segmental instability if the difference between the slip distance on the standing flexion view was greater or equal to $3 \mathrm{~mm}$ compared to the slip distance on the standing extension view ${ }^{6}$. However, this method has been questioned because

Table 2. Comparison of preoperative and postoperative clinical and radiographic assessment

\begin{tabular}{lccc}
\hline & Preoperative & Postoperative & $p$-value \\
\hline VAS scores of back pain & $5.0 \pm 1.3$ & $1.7 \pm 1.2$ & $<0.001$ \\
VAS scores of leg pain & $7.4 \pm 2.0$ & $1.5 \pm 1.6$ & $<0.001$ \\
ODI scores & $49.2 \pm 16.7$ & $17.9 \pm 8.0$ & $<0.001$ \\
Facet joint effusion (mm) & $2.4 \pm 1.0$ & - & - \\
Slip percentage (\%) & $5.3 \pm 20.1$ & $6.8 \pm 21.0$ & 0.613 \\
Segmental motion (mm) & $1.5 \pm 1.1$ & $1.8 \pm 1.0$ & 0.445 \\
\hline
\end{tabular}

VAS, visual analogue system; ODI, Oswestry disability index.

Table 3. Correlation between preoperative radiographic parameters and postoperative clinical outcomes

\begin{tabular}{lccc} 
& Facet joint effusion & Slip percentage & Segmental motion \\
\hline \hline VAS scores of back pain & 0.317 & 0.451 & 0.066 \\
VAS scores of leg pain & 0.159 & 0.588 & 0.582 \\
ODI scores & 0.054 & 0.620 & 0.103 \\
\hline
\end{tabular}

VAS, visual analogue system; ODI, Oswestry disability index. 
of measurement errors, lack of definition of normal movements, and low repeatability, unless the measured vertebral slip was more than $5 \mathrm{~mm}^{1,7}$.

After the first description by Caput et al., facet joint effusion has been regarded as a sign of lumbar segmental instability ${ }^{2}$. Rhin et al. have reported a close linear association between the facet fluid index and the amount of radiographic instability at L4-L5 and concluded that the facet joint effusion on MRI should raise high suspicion of lumbar instability ${ }^{19)}$. Cho et al. have also reported a linear correlation between the degree of segmental motion seen on SLFE radiography and the amount of facet fluid on MR images in patients with degenerative spondylolisthesis at L4-5 $5^{3 \text { ) }}$ One study has even showed that facet fluid is a sensitive predictor of micro-instability in patients with normal dynamic lumbar film ${ }^{12)}$.

However, there is little report on how facet joint effusion affects the outcome of decompression for DLSS without radiographic instability. Tamai et al. have reported that facet joint effusion is observed in $61 \%$ of DLSS patients without radiographic instability and that facet joint effusion has no effect on surgical outcome ${ }^{20)}$. Pao et al. have reported that facet joint effusion observed in MRI taken 6 months after minimally invasive decompression of DLSS without radiographic instability has no effect on clinical or radiographic outcomes ${ }^{16}$. These results are consistent with our findings showing that facet joint effusion without radiographic instability does not affect the clinical or radiological outcomes of decompression for DLSS.

DLSS posterior decompression has many surgical methods with various advantages. However, there is no objective evidence showing that any method is superior ${ }^{15}$. There is a common agreement that preserving facet joints during surgery is very important in preventing postoperative spinal instability ${ }^{8,9)}$. We also made great efforts to preserve the facet joint during surgeries. We believe that this effort plays an important role in preventing postoperative segmental instability in this study.

The addition of spinal fusion to decompression for DLSS inevitably increases the extent of surgery, operation time, blood loss, and in-hospital stay. Fusion surgery is associated with an increased risk of severe complications in elderly patients ${ }^{4}$. In this respect, spinal fusion should be avoided as much as possible in elderly patients who have poor general condition and serious comorbidity. Paradoxically, in order to avoid unnecessary spinal fusion, surgeons must know exactly whether spinal fusion is necessary. Further studies should be attempted to develop more reliable and clear indications of addition of spinal fusion for DLSS patients.

\section{CONCLUSION}

In the absence of segmental instability on SLFE radiographs, facet joint effusion has no effect on clinical or radiographic outcomes of decompression DLSS at L4-L5 level. Facet joints effusion is associated with postoperative slip percentage. However it does not affect clinical outcomes.

\section{REFERENCES}

1. Cabraja M, Mohamed E, Koeppen D, Kroppenstedt S: The analysis of segmental mobility with different lumbar radiographs in symptomatic patients with a spondylolisthesis. Eur Spine J 21: 256-261, 2012

2. Chaput C, Padon D, Rush J, Lenehan E, Rahm M: The significance of increased fluid signal on magnetic resonance imaging in lumbar facets in relationship to degenerative spondylolisthesis. Spine (Phila Pa 1976) 32:1883-1887, 2007

3. Cho BY, Murovic JA, Park J: Imaging correlation of the degree of degenerative L4-5 spondylolisthesis with the corresponding amount of facet fluid. J Neurosurg Spine 11:614-619, 2009

4. Deyo RA, Mirza SK, Martin BI, Kreuter W, Goodman DC, Jarvik JG: Trends, major medical complications, and charges associated with surgery for lumbar spinal stenosis in older adults. JAMA 303: 1259-1265, 2010

5. Eck JC, Sharan A, Ghogawala Z, Resnick DK, Watters WC, 3rd, Mummaneni PV, et al.: Guideline update for the performance of fusion procedures for degenerative disease of the lumbar spine. Part 7: lumbar fusion for intractable low-back pain without stenosis or spondylolisthesis. J Neurosurg Spine 21:42-47, 2014

6. Even JL, Chen AF, Lee JY: Imaging characteristics of "dynamic" versus "static" spondylolisthesis: analysis using magnetic resonance imaging and flexion/extension films. Spine J 14:1965-1969, 2014

7. Frobin W, Brinckmann P, Leivseth G, Biggemann M, Reikeras O: Precision measurement of segmental motion from flexion-extension radiographs of the lumbar spine. Clin Biomech (Bristol, Avon) 11:457-465, 1996

8. Fujiwara Y, Manabe H, Sumida T, Izumi B, Nakanishi K, Tanaka $\mathrm{N}$, et al.: Facet preserving technique by en bloc flavectomy in microscopic posterior decompression surgery for lumbar spinal stenosis: semicircumferential decompression (SCD). Clin Spine Surg 30:197-203, 2017

9. Hatta Y, Shiraishi T, Sakamoto A, Yato Y, Harada T, Mikami Y, et al.: Muscle-preserving interlaminar decompression for the lumbar spine: a minimally invasive new procedure for lumbar spinal canal stenosis. Spine (Phila Pa 1976) 34:E276-280, 2009

10. Herkowitz HN, Kurz LT: Degenerative lumbar spondylolisthesis with spinal stenosis. A prospective study comparing decompression with decompression and intertransverse process arthrodesis. J Bone Joint Surg Am 73:802-808, 1991

11. Iguchi T, Kurihara A, Nakayama J, Sato K, Kurosaka M, Yamasaki K: Minimum 10-year outcome of decompressive laminectomy for degenerative lumbar spinal stenosis. Spine (Phila Pa 1976) 25: 1754-1759, 2000

12. Landi A, Gregori F, Mancarella C, Maiola V, Maccari E, Marotta $\mathrm{N}$, et al.: Lumbar spinal degenerative "microinstability": hype or hope? Proposal of a new classification to detect it and to assess surgical treatment. Eur Spine J 24 Suppl 7:872-878, 2015

13. Mardjetko SM, Connolly PJ, Shott S: Degenerative lumbar spondylolisthesis. A meta-analysis of literature 1970-1993. Spine (Phila Pa 1976) 19:2256s-2265s, 1994

14. Ochia RS, Inoue N, Renner SM, Lorenz EP, Lim TH, Andersson 
GB, et al.: Three-dimensional in vivo measurement of lumbar spine segmental motion. Spine (Phila Pa 1976) 31:2073-2078, 2006

15. Overdevest GM, Jacobs W, Vleggeert-Lankamp C, Thome C, Gunzburg R, Peul W: Effectiveness of posterior decompression techniques compared with conventional laminectomy for lumbar stenosis. Cochrane Database Syst Rev: Cd010036, 2015

16. Pao JL, Chen WC, Chang CH, Chen CS, Wang JL: Clinical significance of postdecompression facet joint effusion after minimally invasive decompression for degenerative lumbar spinal stenosis. J Spinal Disord Tech 27:E318-323, 2014

17. Resnick DK, Watters WC, 3rd, Mummaneni PV, Dailey AT, Choudhri TF, Eck JC, et al.: Guideline update for the performance of fusion procedures for degenerative disease of the lumbar spine. Part 10: lumbar fusion for stenosis without spondylolisthesis. J Neurosurg Spine 21:62-66, 2014
18. Resnick DK, Watters WC, 3rd, Sharan A, Mummaneni PV, Dailey AT, Wang JC, et al.: Guideline update for the performance of fusion procedures for degenerative disease of the lumbar spine. Part 9: lumbar fusion for stenosis with spondylolisthesis. J Neurosurg Spine 21:54-61, 2014

19. Rihn JA, Lee JY, Khan M, Ulibarri JA, Tannoury C, Donaldson $\mathrm{WF}, 3^{\text {rd }}$, et al.: Does lumbar facet fluid detected on magnetic resonance imaging correlate with radiographic instability in patients with degenerative lumbar disease? Spine (Phila Pa 1976) 32: 1555-1560, 2007

20. Tamai K, Kato M, Konishi S, Matsumura A, Hayashi K, Nakamura $\mathrm{H}$ : Facet effusion without radiographic instability has no effect on the outcome of minimally invasive decompression surgery. Global Spine J 7:21-27, 2017 